\title{
A novel excisional wound pain model for evaluation of analgesics in rats
}

\author{
Sergio Parra*, Vaidehi J. Thanawala*, Ajay Rege, and Heather Giles
}

Vapogenix Inc., Houston, TX, USA

Received August 26, 2020

Revised October 27, 2020

Accepted November 3, 2020

Handling Editor: Jong Yeon Park

\section{Correspondence}

Sergio Parra

Vapogenix Inc., 8285 El Rio St, Suite

170, Houston, TX 77054, USA

Tel: +1-713-748-3903 x 227

Fax: $+1-800-779-2906$

E-mail: Protean1974@yahoo.com

* These authors contributed equally to this work.
Background: Management of pain from open wounds is a growing unmet healthcare need. However, the models available to study pain from wounds or to develop analgesics for the patients suffering from them have primarily relied on incisional models. Here, we present the first characterized and validated model of open wound pain.

Methods: Unilateral full-skin excisional punch biopsy wounds on rat hind paws were evaluated for evoked pain using withdrawal responses to mechanical and thermal stimulation, and spontaneous pain was measured using hind paw weight distribution and guarding behavior. Evaluations were done before wounding (baseline) and 2-96 hours post-wounding. The model was validated by testing the effects of buprenorphine and carprofen.

Results: Pain responses to all tests increased within 2 hours post-wounding and were sustained for at least 4 days. Buprenorphine caused a reversal of all four pain responses at 1 and 4 hours post-treatment compared to $0.9 \%$ saline $(P<0.001)$. Carprofen decreased the pain response to thermal stimulation at $1(P \leq 0.049)$ and 4 hours $(P<0.011)$ post-treatment compared to $0.9 \%$ saline, but not to mechanical stimulation.

Conclusions: This is the first well-characterized and validated model of pain from open wounds and will allow study of the pathophysiology of pain in open wounds and the development of wound-specific analgesics.

Key Words: Analgesics; Biopsy; Hyperalgesia; Models, Animal; Nociceptive Pain; Pain Management; Pain, Procedural; Rats; Wound and Injuries.

\section{INTRODUCTION}

Almost 165 million people worldwide suffer from wounds. Of these, more than 60 million people have wounds of non-surgical origins, including lacerations, burns, and chronic ulcers (diabetic, venous, pressure) [1]. Severe pain from acute and chronic wounds is widespread, but is frequently underappreciated and poorly treated. For open wounds, patients report pain as the worst problem, which is severe in $70 \%$ of the cases $[2,3]$. Wound pain management is medically challenging because it requires longterm prescription of analgesics, as well as acute control of its exacerbation during routine wound care procedures such as dressing changes, cleansing, and debridement of necrotic tissue $[4,5]$.

Current treatment for wound pain relies heavily on systemic pain medications, mainly oral nonsteroidal antiinflammatory drugs (NSAIDs) and opioids [5,6]. These op- (c) This is an open-access article distributed under the terms of the Creative Commons Attribution Non-Commercial License (http://creativecommons.org/licenses/by-nc/4.0/), which permits unrestricted non-commercial use, distribution, and reproduction in any medium, provided the original work is properly cited.

(C) The Korean Pain Society, 2021
Author contributions: Sergio Parra: Writing/manuscript preparation, Study conception, Data acquisition, Data analysis; Vaidehi J. Thanawala: Writing/manuscript preparation, Study conception, Data acquisition, Data analysis; Ajay Rege: Writing/manuscript preparation; Heather Giles: Writing/manuscript preparation. 
tions frequently provide inadequate pain control and often result in intolerable systemic adverse effects [4]. NSAIDs have numerous drawbacks, including risk of bleeding, gastric, renal, and cardiac complications $[7,8]$. These adverse events are especially challenging for older patients that mostly comprise the chronic open wounds population. Opioid drugs are also associated with numerous problems, including cognitive impairment, constipation, tolerance, addiction, abuse, and at high doses, respiratory depression. Opioid addiction and deaths due to overdose in the United States (US) have reached epidemic proportions, leading to a National Public Health Emergency in 2017 [9]. Therefore, there exists an unmet need for nonopioid, alternative analgesic options for wound pain.

Currently, EMLA is the only topical analgesic with a robust effect to prevent pain due to wound debridement $[10,11]$, but it requires a long application time, which is impractical for most wound care services. Several clinical studies have also shown local pain relief in patients with open wounds following the use of an ibuprofen-releasing foam dressing, however, the overall degree of acute analgesia was modest $[12,13]$. Thus, there is an imminent need to develop faster acting, effective analgesics for open wounds.

The lack of options for adequate management of pain associated with wounds, especially open wounds, may be the result of a lack of appropriate models to test potential candidates. Previously, animal models in rats and pigs were developed to characterize the mechanisms of pain responses following a surgical incision and to investigate novel treatments for post-operative wound pain [14-16]. Recently, rat models have been developed to investigate the pain mechanisms of burns $[17,18]$, and evaluate treatment modalities [17]. However, the investigation of analgesics to treat pain from open wounds has not made much progress in decades.

The aim of this study was to develop, characterize, and validate a rat model of excisional wound pain. This new model is characterized by spontaneous pain behaviors as well as enhanced responses to evoked pain through mechanical and thermal stimulation. This model will be useful to facilitate the investigation of pain mechanisms in open wounds and for the testing of novel analgesic treatments.

\section{MATERIALS AND METHODS}

\section{Animals}

The experiments were conducted with an equal number of 200-400 g male and female Sprague Dawley rats from En- vigo (Indianapolis, IN). Six to eight animals were used per group for all experiments as described in the respective section for a total of 40 animals. The animals were housed in temperature and humidity-controlled conditions with a 12-hour light/dark cycle and had free access to food and water. The animals were kept on Certified Irradiated Diamond Soft bedding (7989C, Envigo) to provide a soft bedding especially after wounding. The study protocol and all the procedures were ethically reviewed and approved by the local Institutional Animal Care and Use Committee of Nanospectra Biosciences, Inc. (D16-00790 [A4560-01]), adhered to the Guide for the Care and Use of Laboratory Animals, the Research Council's Guide for the Care and Use of Laboratory Animals, and the US Public Health Service's Policy on Humane Care and Use of Laboratory Animals, and complied with Animal Research: Reporting of In Vivo Experiments (ARRIVE) guidelines.

The wound pain model was characterized by evaluating evoked pain: responses to thermal stimulation, mechanical stimulation, and spontaneous pain: hind paw weight distribution (HPWD) and guarding behavior. Eight (4 males and 4 females) rats were used for the thermal stimulation and HPWD tests and another set of 8 animals was used for the mechanical stimulation and guarding behavior tests. Therefore, a total of 16 animals were used for the model characterization. Animals were assigned to the study groups using a random sequence. An increase in pain score is indicated by a decrease in the time to withdrawal from thermal stimulation, decrease in force to withdrawal from mechanical stimulation, increased guarding behavior, and decreased weight bearing on the injured paw compared to the uninjured paw.

\section{Experimental timeline}

Animals were acclimated for 72 hours after receipt. On day -2 , after acclimation, the animals were trained on the instruments, followed by baseline measurements on day -1 . On day 0 , they received a punch biopsy as described below and were tested once daily for the spontaneous and evoked pain responses on days 1-4, followed by euthanasia on day 4. For all the testing procedures, the rats were acclimated to the testing room for 20-30 minutes and to the thermal stimulation and mechanical stimulation instruments for 15-20 minutes. During the acclimation to the testing room, the animals had free access to food and water.

\section{Punch biopsy}

The animals were anesthetized with isoflurane (5\% for induction and $2 \%-3 \%$ for maintenance) by using a low flow anesthesia delivery system (SomnoSuite ${ }^{\circledR}$; Kent Scientific, 


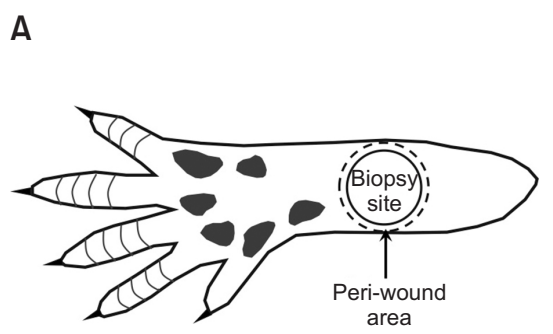

B

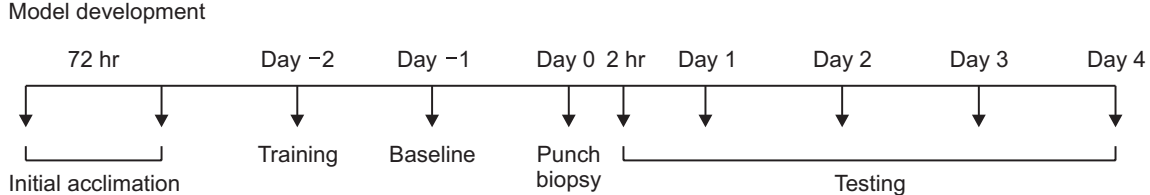

Initial acclimation

Model validation



Fig. 1. (A) Schematic of the plantar side of the rat hind paw showing the punch biopsy site ( $5 \mathrm{~mm}$ diameter) and peri-wound stimulation areas. (B) Timelines for development (upper panel) and validation (lower panel) experiments in the excisional wound pain model in rats. Excisional wounds were generated by punch biopsy of the skin of the hind paw, followed by pain behavioral testing at different time points. The rats used for the validation experiments were also treated with carprofen or buprenorphine in a crossover manner. ${ }^{a}$ Saline on day 1 and buprenorphine on day 3 or carprofen on day 1 and saline on day 3.

Torrington, CT). The left hind paw was prepped with povidone iodine swab sticks and alcohol wipes $(\times 3)$. A hole was made in the sterile drape and the paw was pulled through the hole so adjacent contaminated parts of the animal were covered. A $5.0 \mathrm{~mm}$ sterile skin biopsy punch (RBP50; Robbins Instruments, Chatham, NJ) was used to apply pressure and twisted in a drilling motion until the blade of the skin punch pierced the epidermis of the skin. The precise site of biopsy was between the walking pads and the proximal edges of the heel of the hind-paw (Fig. 1A). The skin punch was removed after the blade had sufficiently carved out a cylinder of skin. The dermis of the cored skin was grabbed with forceps and using the scissors the cored skin was cut free. Excess blood was wiped off using a sterile gauze and the rat was returned to a clean cage with soft bedding.

\section{Mechanical stimulation}

The rats were acclimated to the testing environment by placing on a $1.1 \mathrm{~cm}^{2}$ mesh base platform in an acrylic animal enclosure $(8 \times 3.5 \times 3.5 \mathrm{in})$ for 20-30 minutes. Von Frey filaments of 1.4, 2, 4, 6, 8, 10, 15, 26, and $60 \mathrm{~g}$ (Aesthesio ${ }^{\circledR}$; DanMic Global LLC, San Jose, CA) were applied to the rat paw in ascending order. Filaments were applied from the underside of the mesh to the plantar surface (wound and peri-wound, Fig. 1A) of the rat's hind paw, presented perpendicular to the rat paw and applied with enough force to cause a slight bend in the filament. The process was repeated with increasing filament weights until stimulation elicited a hind paw withdrawal. At threshold, the rat responded by flicking its paw away from the stimulus. The mechanical withdrawal threshold was defined as the minimum filament force that elicited a withdrawal reflex. Once a response was noted, no further weights were applied to that region/paw. Each area was tested 4 times, with each set of stimulations at least a minute apart to avoid any sensitization or wind-up effects. Baselines were recorded on day -1 and testing was conducted as indicated (Fig. 1B) after the punch-biopsy or wounding. Most animals had developed a hard scab at the wound site within 2-24 hours, making it difficult to obtain a response in the wound beyond those time points. Therefore, the periwound area was also tested as indicated in Fig. 1A.

\section{Thermal stimulation}

Thermal stimulation was applied to the plantar surface of the animal paws using a heat source under a glass platform. The rats were acclimated to the testing environment by placing them on the glass surface in an acrylic animal enclosure $(8 \times 3.5 \times 3.5$ in) for 20-30 minutes. Baselines were recorded on day -1 and testing was conducted as indicated (Fig. 1B) after the punch-biopsy or wounding. After acclimation, the infrared generator beam was adjusted using the guide until it was under the hind paw, below the wound (Fig. 1A, 2A) and the heat source was initiated. The paw withdrawal latency was recorded by the Plantar Test thermal stimulator (Hargreaves Apparatus; Ugo Basile, Gemonio, Italy) when the animal lifted the paw in response to the heat stimulus. Each paw was tested 3 times, with consecutive stimulations at least a minute apart to avoid any sensitization or wind-up effects. The thermal stimulation lasted a maximum of 20.1 seconds to ensure there was no injury to the animal. 

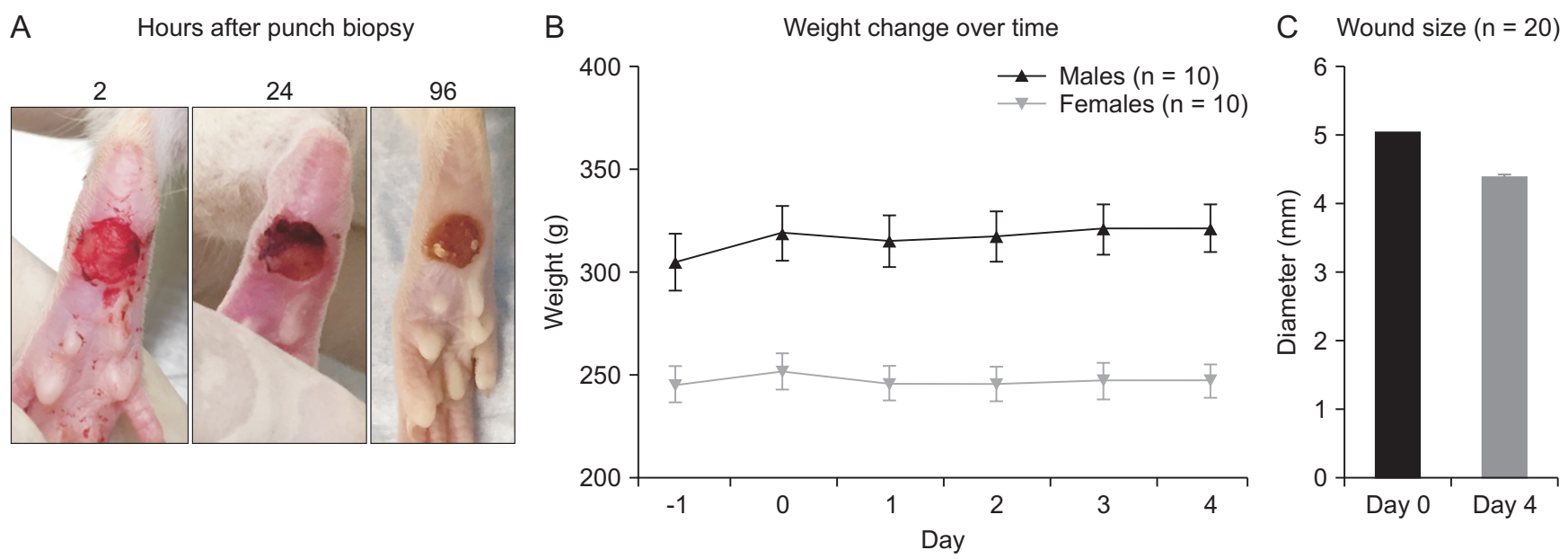

Fig. 2. (A) Representative images of the state of the excisional wound at 2, 24 , and 96 hours after punch biopsy. (B) Average body weight over the course of the experiment ( $n=20$ rats per group). (C) Average wound size change from day of biopsy to end of study ( $n=40$ rats total). The error bars indicate standard error of the mean.

\section{Guarding behavior}

The guarding behavior measurement method was adapted from Brennan et al. [14]. Animals were allowed to move freely on a mesh base. An investigator recorded the guarding behavior as pain scores that represented the amount of weight the rat exerted on the injured paw (or to-be injured paw for baseline recording): full weight (0), partial weight with no blanching (1), completely off (2). Each rat was observed for 1 minute every 6 minutes for 1 hour and the sum of the scores over the entire hour (total of 10 scores per rat) was recorded as the guarding behavior for each rat at that time point.

\section{HPWD}

Weight-bearing by the injured and uninjured hind paws was compared using a static weight-bearing instrument. Animals were acclimated to the rat enclosure of the Static Weight Bearing-Touch instrument (BIO-SWB-TOUCH-R; Bioseb, Pinellas Park, FL), and the operator ensured that the rat held the front paws on the incline and that each hind paw rested on the base weighing plates. Once the rats were acclimated, the weight exerted on each hind paw was recorded over 10 seconds. It was ensured that the rat stayed relatively stationary throughout the entire recording time. If there were any major movements, the readout was disregarded and repeated. HPWD for each animal was recorded 6 times for the model development and 3 times for the testing of buprenorphine.

\section{Model validation and drug administration}

For validation of this pain model, two known systemic analgesics were tested: carprofen and buprenorphine. The baseline pain evaluations were made before wounding and on day 1 , after wounding. The investigator conducting the evaluation was blinded to the drugs administered. Six rats ( 3 males and 3 females) per group were then administered $5 \mathrm{mg} / \mathrm{kg}$ carprofen (Carprieve ${ }^{\circledR}$; Norbrook, Newry, Northern Ireland), $0.1 \mathrm{mg} / \mathrm{kg}$ buprenorphine (Simbadol ${ }^{\circledR}$; Zoetis, Parsippany, NJ) or saline subcutaneously on the animals' backs, and the responses to the different tests in the model were recorded prior to and at 1,4 , and 24 hours post-drug administration. Twelve animals were used for mechanical stimulation and guarding behavior tests and 12 animals were used in the thermal stimulation and HPWD tests. Therefore, a total of 24 animals were used in the model validation. The doses for carprofen and buprenorphine were selected based on previous reports [19-22]. Due to the scab formation, only the peri-wound site (Fig. 1A) was tested for the mechanical stimulation. A crossover design was implemented to reduce the number of animals used. One group of animals received carprofen on day 1 and saline on day 3 ; another group received saline on day 1 and buprenorphine on day 3 (Fig. 1B). Temporal groups were compared for analyses.

\section{Statistical analysis}

For all the rat experiments, sample size calculations used a power of $0.8-0.9$, a $P=0.05$, and a Cohen effect size (f) $=$ 0.6 [23]. The power analysis showed that $6-8$ rats per group were sufficient to demonstrate statistical significance, assuming multiple groups with at least 4 measurements over time (repeated measures design). All the calculations were done with the software G*Power Version 3.1.

Data are presented as group mean \pm standard error of 
means. One-way and two-way analysis of variance, followed by Sidak's or Tukey's post-hoc tests were used to analyze all the pain behaviors. Values of $P<0.05$ were considered significant. Analyses were done using GraphPad Prism version 6.0h for Mac OS X (GraphPad Software Inc., San Diego, CA).

\section{RESULTS}

Rats groomed normally and appeared to be in a good state of welfare during the experimental period. There was a small decrease in body weight ( $<2 \%$ on average) for both males and females the first day after wounding. Subsequently, the weights remained stable or increased (Fig. 2B).
Healing was progressive, and the diameter of the wound decreased from $5 \mathrm{~mm}$ to $4.35 \mathrm{~mm}$ (range: $3.11-5.08 \mathrm{~mm}$ ) at the end of the observation period, 4 days after wounding (Fig. 2C).

\section{Excisional wound pain model development}

\section{1) Mechanical stimulation}

There was no difference in the baseline values for the mechanical von Frey force required for withdrawal between the two hind paws of the animals $(\mathrm{n}=8)$. After punch biopsy, the force to withdrawal for the injured paw was different for the uninjured paw at 2, 24, and 48 hours ( $P$ $<0.001, P \leq 0.049$, and $P<0.011$, respectively) (Fig. 3A).
A

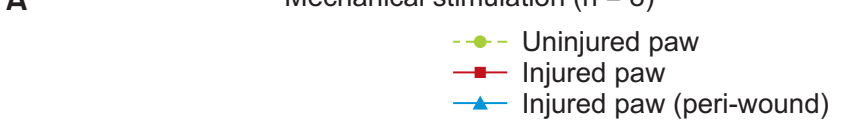

B

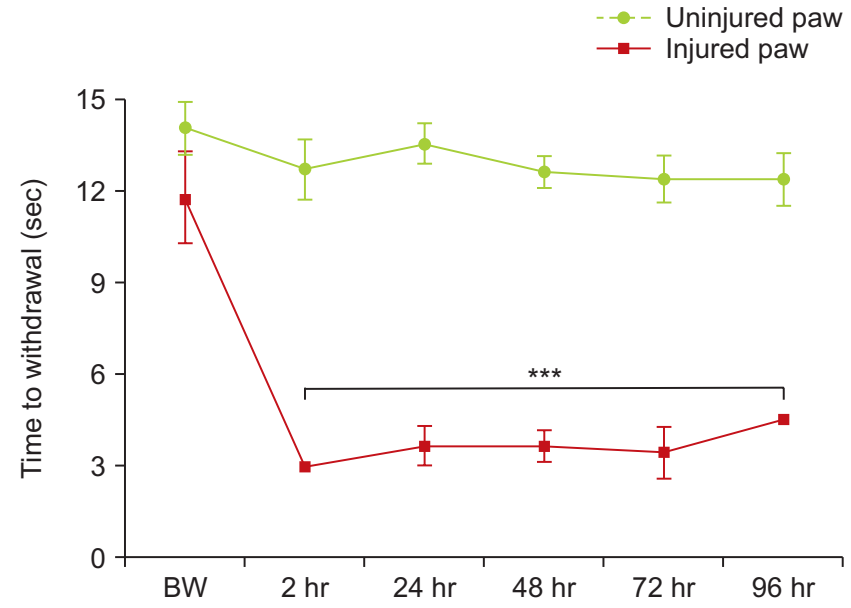

D

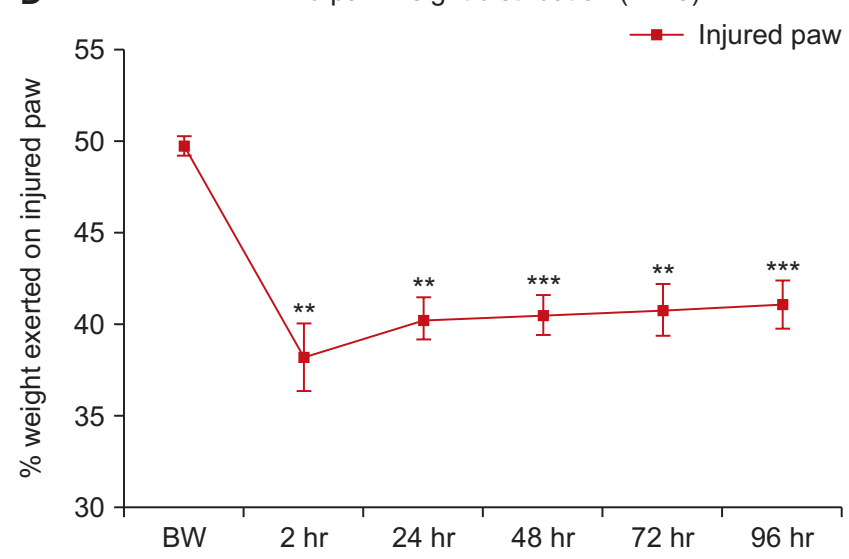

Fig. 3. Responses at baseline and 2-96 hours post punch biopsy for injured and uninjured paw. (A) Force to withdrawal using von Frey filaments (including peri-wound area). Statistical comparisons were done using ordinary two-way analysis of variance (ANOVA) followed by Sidak's post-hoc test (a repeated measures approach was not possible due to two missing values). (B) Time to withdrawal during thermal stimulation. Statistical comparisons were done using repeated-measures two-way ANOVA followed by Sidak's post-hoc test. (C) Guarding behavior for the injured paw. (D) Percentage of body weight exerted on injured paw vs. uninjured paw. Statistical comparisons for $(C)$ and (D) were done using repeated-measures one-way ANOVA followed by Sidak's post-hoc test. $\mathrm{N}=8$ rats per group. The error bars indicate standard error of the mean. BW: before wounding. * ***** $P \leq 0.049,0.011,0.001$, respectively. 

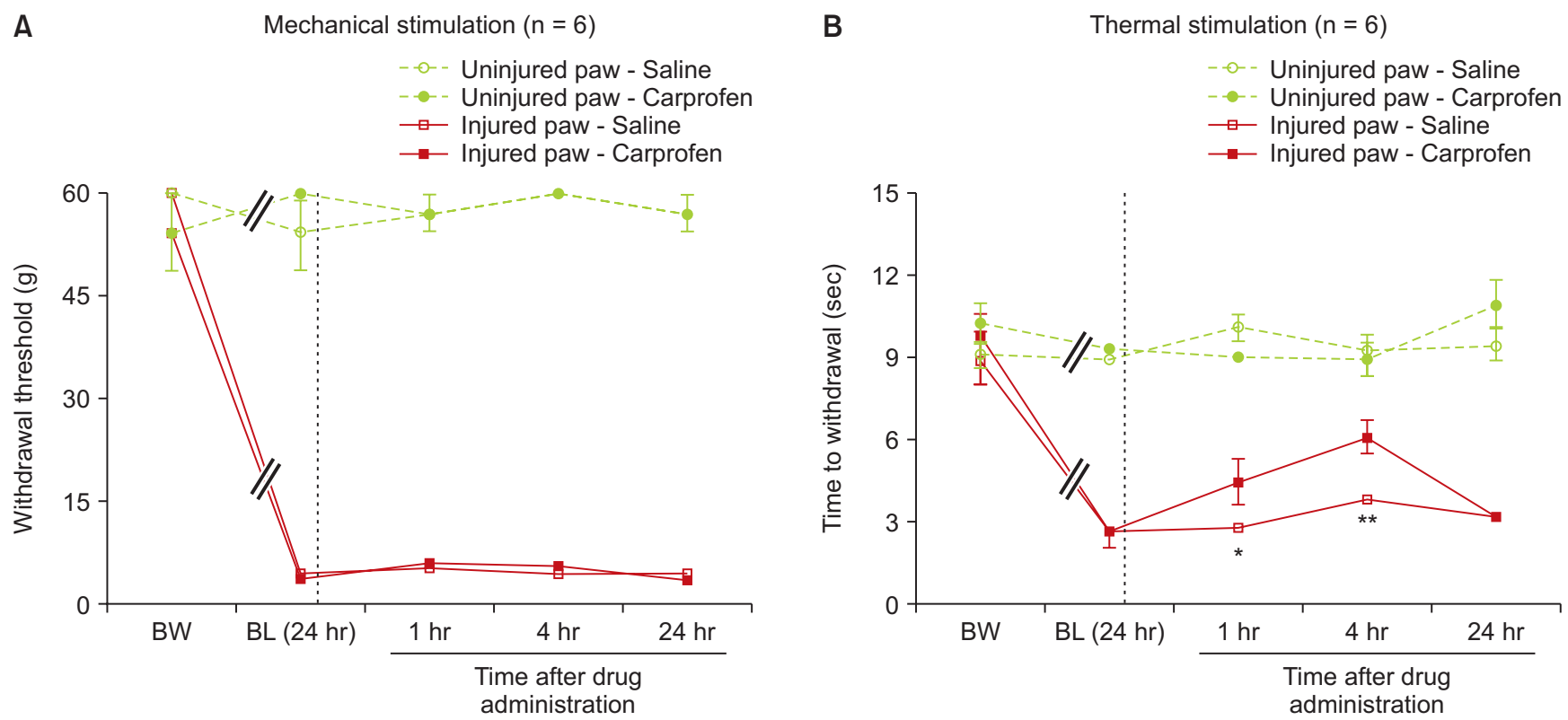

Fig. 4. The effect of a single dose of carprofen (solid) or saline (open) on responses to von Frey (A) and thermal stimulation (B) in the injured (red) and uninjured (green) paws (dosing 24 hours post punch biopsy). Statistical comparisons were done using repeated-measures two-way analysis of variance followed by Sidak's post-hoc test. The dotted line inside the graph indicates the time of drug administration. $\mathrm{N}=6$ rats per group. The error bars indicate standard error of the mean. BW: before wounding, BL (24 hr): baseline at 24 hours after wounding. *** $P \leq 0.049$, and 0.011, respectively.

Most animals had developed a hard scab at the wound site within 2-24 hours (Fig. 2A), making it difficult to obtain a response in the wound beyond those time points. Therefore, the peri-wound area was also tested. The force to withdrawal for the peri-wound area on the injured paw was less than the uninjured paw at 2, 24, 48, 72, and 96 hours $(P<0.001)$ (Fig. 3A).

\section{2) Thermal stimulation}

There was no difference in the baseline values for time to withdrawal following thermal stimulation between the two hind paws $(\mathrm{n}=8)$. After punch biopsy, the time to induce withdrawal in the injured paw was shorter at 2, 24, 48,72 , and 96 hours compared with the uninjured paw ( $P$ $<0.001$ ) (Fig. 3B).

\section{3) Guarding behavior}

Before punch biopsy, the animals showed minimal to no guarding behavior $(\mathrm{n}=8)$. All animals showed a consistent increase in guarding behavior of the injured paw at 2, 24, 48, $72(P<0.001)$, and 96 hours $(P<0.001)$ as compared to the baseline before wounding (Fig. 3C).

\section{4) HPWD}

At baseline before punch biopsy, the animals exerted equal weight $(50 \%)$ on both hind paws $(n=8)$. After punch biopsy, the animals put significantly less weight on the injured paw at 2, $24(P<0.01), 48(P<0.001), 72(P<0.011)$, and 96 hours $(P<0.001)$ as compared to the baseline before wounding, showing an altered weight distribution between the hind paws (Fig. 3D).

\section{Validation of the excisional wound model}

\section{1) Effect of carprofen on evoked pain responses}

Carprofen increased the time to paw withdrawal following thermal stimulation on the injured paw at $1(P<0.05)$ and 4 hours $(P<0.01)$ after administration (Fig. $4 \mathrm{~A})(\mathrm{n}=6)$. At 24 hours after carprofen administration, the response to thermal stimulation returned back to baseline. However, carprofen did not change the response to mechanical stimulation (Fig. 4B). In the uninjured paw, the pain responses to mechanical or thermal stimulation remained unchanged after carprofen administration.

2) Effect of buprenorphine evoked and non-evoked pain responses

All rats showed an increase in baseline pain score 72 hours after wounding on the injured paw, compared to the baseline before wounding. There was no change in the pain score of the uninjured paw before or after wounding.

Buprenorphine increased the withdrawal thresholds to mechanical or thermal stimulation on the injured paw 

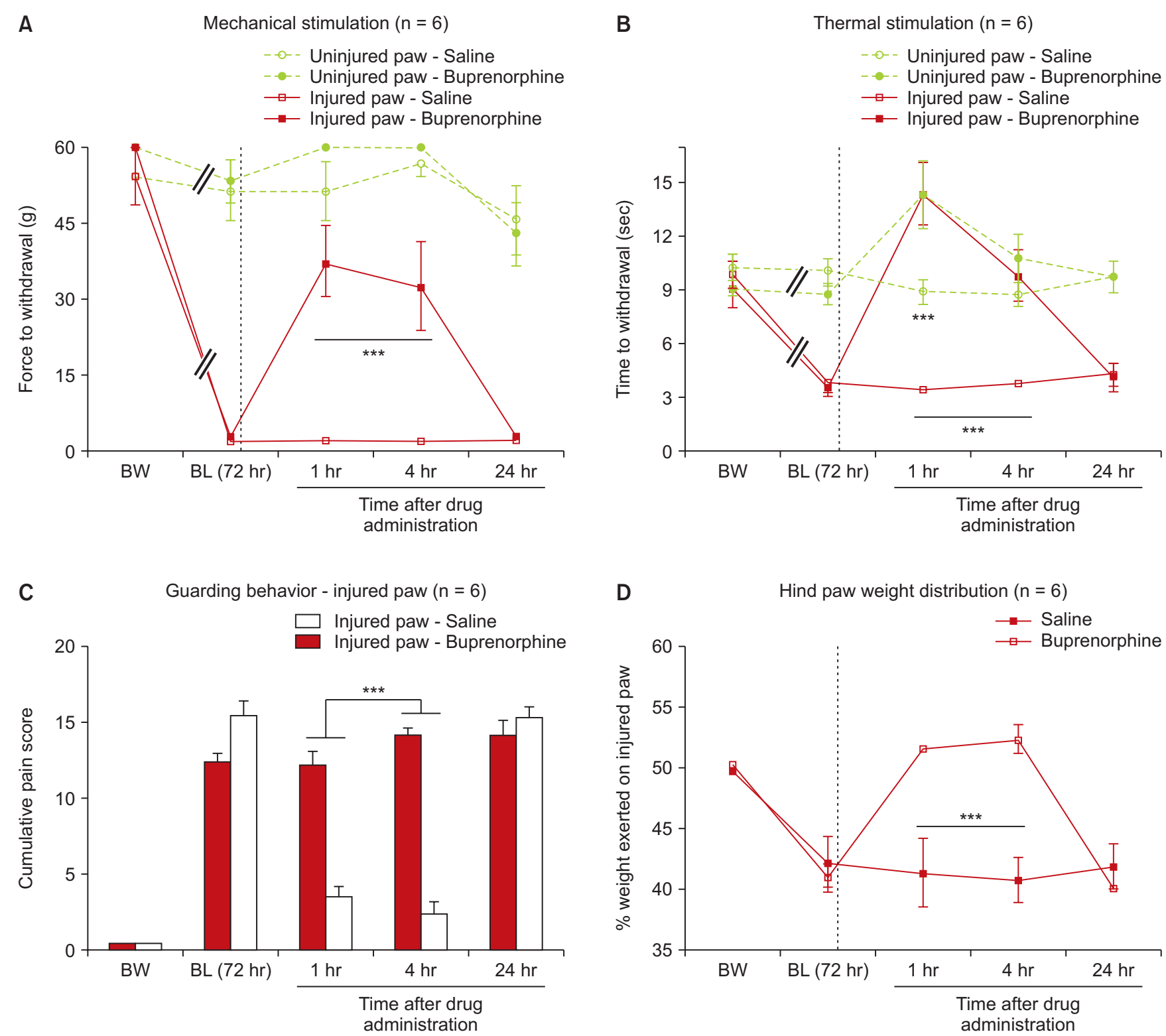

Fig. 5. The effect of a single dose of buprenorphine (solid) or saline (open) on responses to von Frey (A) and thermal stimulation (B) in the injured (red) and uninjured (green) paws (C) guarding behavior for the injured paw and (D) percentage of body weight exerted on injured paw vs. uninjured paw (dosing 72 hours post punch biopsy). Statistical comparisons were done using repeated-measures two-way analysis of variance followed by Tukey's post-hoc test for $A$ and B and followed by Sidak's post-hoc test for $(C)$ and $(D)$. The dotted line inside the graph indicates the time of drug administration. $N=6$ rats per group. The error bars indicate standard error of the mean. BW: before wounding, BL (72 hr): baseline at 72 hours after wounding. $* * * P<0.001$.

at 1 and 4 hours $(P<0.001)$ after administration $(n=6)$. At 1-hour post-administration, buprenorphine also increased the time to induce withdrawal to thermal stimulus on the uninjured paw $(P<0.001)$ (Fig. 5A, B). Buprenorphine reduced the guarding behavior and restored the weight exerted on the injured paw at 1 and 4 hours $(P<0.001)$ after administration (Fig. 5C, D). At 24 hours post buprenorphine administration, the pain behaviors returned to baseline after wounding.

\section{DISCUSSION}

Pain from open wounds is a widespread and growing concern. Chronic wounds are an increasing healthcare burden, due to the rapidly rising incidence of diabetes and obesity, and the growing elderly population [24]. Traumatic wounds, often cared for in the emergency department [25], can result in severe pain as well. However, wound pain is a poorly managed problem and is frequently treated with systemic medications which have multiple undesirable effects. A major purpose of this study was to develop an excisional wound pain model that can evaluate 
evoked and spontaneous pain behaviors and be useful for the evaluation of new analgesics for painful open wounds. A punch biopsy was used to create a full-thickness excisional wound, which was left open to start healing by secondary intention. The excisional wound resulted in rapid, robust, stable, and long-lasting pain responses for at least 96 hours after wounding and permitted the detection of analgesia after systemic drug administration. To the best of our knowledge, this is the first excisional, full-thickness, open wound pain animal model that has been extensively characterized and validated.

We chose Sprague Dawley rats to develop the model because they are commonly used for behavioral studies. Currently available techniques and instrumentation restrict some pain behavior testing depending on injury location. Rats have a sufficient plantar paw size that allows wounding and permits evaluation of both evoked and spontaneous pain, in contrast to using other animal body areas (e.g., back [18]). Our studies used a 5-mm punch biopsy to create a wound big enough for testing, but not so large as to cause significant discomfort to the animal. After the punch biopsy, the animals were evaluated for evoked pain behaviors (mechanical and thermal stimulations) and for spontaneous pain behaviors (HPWD and guarding behavior). As expected, the injured paw was more sensitive to the von Frey filaments compared to the uninjured paw or the prewounding baseline and that this sensitivity lasts at least for 96 hours. Interestingly, the peri-wound area remained more sensitive to the mechanical stimulation compared to the wound itself. This was probably due to the formation of a hard scab on the wound surface which starts to form between 2 to 24 hours post-wounding. In the thermal stimulation test, the primary wound site on the injured paw was consistently sensitive up to 96 hours compared to the uninjured paw. We hypothesize that mechanical and thermal stimulation of the wound may be representative of any clinical intervention involving the application of physical forces or substances to an open wound (dressing application/removal, debridement or cleaning). Therefore, these tests may have predictive capability to evaluate factors that worsen or alleviate acute exacerbation of wound pain due to wound care procedures.

With regard to spontaneous pain behaviors, paw licking and the grimace scale were initially assessed, but the changes observed were inconsistent and small, and therefore, further evaluation was not conducted. Both HPWD and guarding behavior tests consistently showed that the animals preferred to bear less weight on the injured paw, which was consistent up to 96 hours. Even though both tests measure paw weight bearing, the HPWD test has some advantages over the guarding behavior test: (a) HPWD is a direct quantitative test and (b) can be done much faster and for multiple time points within an hour compared to the guarding behavior test. The main challenge of using the HPWD test is that the animals need to be well-trained and acclimated to the instrument to ensure stable readings. Under appropriate conditions, the HPWD test provides a reliable indication of spontaneous pain, which is informative and advantageous for the study wound pain at rest or due to posture or locomotion.

A major reason for developing the rat excisional wound pain model was to allow evaluation of analgesic drugs. We validated the model using known systemic analgesics: an opioid, buprenorphine, and an NSAID, carprofen. Buprenorphine is a partial mu-opioid receptor (MOR) agonist with very high affinity for the MOR and low intrinsic efficacy. The dose and route of administration of buprenorphine were based on previously published studies in rats $[21,22]$. A single dose of buprenorphine $(0.1 \mathrm{mg} / \mathrm{kg})$ showed a dramatic decrease in the pain responses in all four tests at the injured paw after 1 and 4 hours compared to the baseline after wounding and even caused a reduction in the sensitivity of the uninjured paw to thermal stimulation compared to baseline, probably due to a higher threshold of pain response conferred by buprenorphine. Carprofen reduced the pain responses to the thermal stimulation on the injured paw, although to a lesser extent than buprenorphine, but it did not have any effect on the pain responses to mechanical stimulation. The difference in the responses to thermal versus the mechanical stimulation may be related to the dose of carprofen used or because the time points selected for measurement were suboptimal. Additional experiments, including HPWD and guarding behavior, will have to be performed to further explore the analgesic profile of carprofen in our model. Carprofen is an NSAID whereas buprenorphine is an opioid. Therefore, it is likely that carprofen has a distinct analgesic profile in our model compared to buprenorphine. It will be interesting to study the analgesics from different classes and the differences in their efficacy in our model of wound pain.

Previously described animal wound pain models $[14,16,17]$ are for incisional wound pain, surgical wound pain or for burns. The most commonly used model is the Brennan model, which involves an incision on the hind paw in the skin and fascia and sometimes muscle, followed by suturing. This model has been widely used for the evaluation of pathophysiological mechanisms of wound pain [26] and post-surgical analgesic options $[27,28]$. The pain behaviors tested in the Brennan model show statistically significant differences only for up to 72 hours postwounding, which is a limitation in evaluating the duration of action of extended-release analgesics $[29,30]$. In addition, the mechanical stimulation testing is done on the area around the wound and not on the actual wound site. 
A similar wound pain model has been developed in pigs, which involves a long incisional wound on the lower back followed by suturing. This wound model is characterized by a rapid, robust, stable, and long-lasting pain response to mechanical stimulation on an area proximal to the wound and not on the actual wound site, but no other pain behaviors have been consistently shown in this particular model [16,31-33]. This model could be useful for the evaluation of topical analgesics since porcine skin is considered to closely resemble human skin. However, studies using pigs are expensive, and using them as the primary model for pain research is not always possible.

Another wound pain model in rats was developed by the US Army Institute of Surgical Research using a full-thickness plantar thermal injury. The pain behaviors characterized in this model are long-lasting, but they are delayed, modest, and present only at the skin sites adjacent to the original point of injury $[17,34]$. More recently, a pain model with open wounds on the back in rats was described. However, the model was not extensively characterized, and the authors only evaluated the exacerbation of pain due to mechanical stimulation after local application of adrenocorticotropic hormone on day 5 post-wounding [18].

Given the limitations of other rat wound pain models (pain behaviors of short duration in incisional wounds or delayed onset in burns), the open wound pain model presents multiple advantages: the possibility of direct and indirect stimulation of the primary site of injury with rapid, robust, stable and long-lasting pain responses. These differential characteristics may be useful for better understanding of open wound pain pathophysiology and development of analgesics aimed at treating wound pain. The open wound model allows testing for evoked (by both thermal and mechanical stimuli) as well as spontaneous pain, with consistent pain responses for at least 96 hours. The testing of evoked pain in our model provides a method to evaluate the potential effect of drugs on the pain experienced by patients during wound care procedures. In addition, the potential effects of analgesics on resting pain, or pain due to movement/and or postural changes can be evaluated using HPWD or guarding behavior. Moreover, no gross sex differences were observed for any of the tests. The major limitation of our model is the application of topical analgesics, since the wound site is located on the paw of the animal, it may require restraining to ensure sufficient contact for a topical analgesic to be tested in this model. Based on the other wound pain models, we evaluated pain responses up to 96 hours, however since we see robust responses even at 96 hours, it may be interesting to explore the actual duration of the pain responses to further characterize the model.

The incisional wound pain models have been instru- mental in the non-clinical characterization of new local analgesic options for the treatment of post-surgical wound pain $[29,31,32]$ (e.g., liposomal bupivacaine [35]). In contrast, there are few options available for pain associated with open wounds. We hypothesize that the development of analgesic options for open wounds will be facilitated by developing and using models that are specific for open wounds. Our excisional wound pain model can be used to study the pathophysiology of pain in open wounds, the mechanism of action of known drugs, and to test novel analgesics. We further anticipate that this model can be developed in other species like pigs. In conclusion, we present, for the first time, an open wound animal pain model with robust, long-lasting pain responses to evoked and spontaneous pain.

\section{ACKNOWLEDGMENTS}

We would like to thank Dr. Marcie Fowler for her input on the initial model development strategy. We also thank Dr. Emilio Lucero for his help reformatting the references.

\section{CONFLICT OF INTEREST}

All authors are (or were) employees of Vapogenix, Inc. No potential conflict of interest relevant to this article is reported.

\section{FUNDING}

All funding for this research was provided by Vapogenix, Inc.

\section{ORCID}

Sergio Parra, https://orcid.org/0000-0002-3191-0475

Vaidehi J. Thanawala, https://orcid.org/0000-0002-4774-5937

Ajay Rege, https://orcid.org/0000-0001-7412-3140

Heather Giles, https://orcid.org/0000-0002-0341-5602

\section{REFERENCES}

1. BioNap. Big changes coming to the wound care market in 2014 [Internet]. Ra'anana: Seeking Alpha; 2013. Available at: http://seekingalpha.com/article/1757332-big-changescoming-to-the-wound-care-market-in-2014.

2. Phillips T, Stanton B, Provan A, Lew R. A study of the impact 
of leg ulcers on quality of life: financial, social, and psychologic implications. J Am Acad Dermatol 1994; 31: 49-53.

3. Hofman D, Ryan TJ, Arnold F, Cherry GW, Lindholm C, Bjellerup M, et al. Pain in venous leg ulcers. J Wound Care 1997; 6: 222-4.

4. Price PE, Fagervik-Morton H, Mudge EJ, Beele H, Ruiz JC, Nystrøm TH, et al. Dressing-related pain in patients with chronic wounds: an international patient perspective. Int Wound J 2008; 5: 159-71.

5. Cooper SM, Hofman D, Burge SM. Leg ulcers and pain: a review. Int J Low Extrem Wounds 2003; 2: 189-97.

6. Noonan L, Burge SM. Venous leg ulcers: is pain a problem? Phlebology 1998; 13: 14-9.

7. Malchow RJ, Black IH. The evolution of pain management in the critically ill trauma patient: emerging concepts from the global war on terrorism. Crit Care Med 2008; 36(7 Suppl): S346-57.

8. Farkouh ME, Greenberg BP. An evidence-based review of the cardiovascular risks of nonsteroidal anti-inflammatory drugs. Am J Cardiol 2009; 103: 1227-37.

9. HHS. HHS Acting Secretary declares public health emergency to address national opioid crisis [Internet]. Washington, D.C.: HHS; 2017. Available at: https://www.hhs.gov/about/ news/2017/10/26/hhs-acting-secretary-declares-publichealth-emergency-address-national-opioid-crisis.html.

10. Rosenthal D, Murphy F, Gottschalk R, Baxter M, Lycka B, Nevin K. Using a topical anaesthetic cream to reduce pain during sharp debridement of chronic leg ulcers. J Wound Care 2001; 10: 503-5.

11. Agrifoglio G, Domanin M, Baggio E, Cao P, Alberti AN, Bonn $F$, et al. EMLA anaesthetic cream for sharp debridement of venous leg ulcers: a double-masked, placebo-controlled study. Phlebology 2000; 15: 81-3.

12. Romanelli M, Dini V, Polignano R, Bonadeo P, Maggio G. Ibuprofen slow-release foam dressing reduces wound pain in painful exuding wounds: preliminary findings from an international real-life study. J Dermatolog Treat 2009; 20: 1926.

13. Gottrup F, Jørgensen B, Karlsmark T, Sibbald RG, Rimdeika R, Harding $\mathrm{K}$, et al. Reducing wound pain in venous leg ulcers with Biatain Ibu: a randomized, controlled double-blind clinical investigation on the performance and safety. Wound Repair Regen 2008; 16: 615-25.

14. Brennan TJ, Vandermeulen EP, Gebhart GF. Characterization of a rat model of incisional pain. Pain 1996; 64: 493-501.

15. Brennan TJ. Postoperative models of nociception. ILAR J 1999; 40: 129-36.

16. Castel D, Willentz E, Doron O, Brenner O, Meilin S. Characterization of a porcine model of post-operative pain. Eur J Pain 2014; 18: 496-505.

17. Fowler M, Clifford JL, Garza TH, Slater TM, Arizpe HM, Novak J, et al. A rat model of full thickness thermal injury char- acterized by thermal hyperalgesia, mechanical allodynia, pronociceptive peptide release and tramadol analgesia. Burns 2014; 40: 759-71.

18. Goto T, Nakagami G, Minematsu T, Tomida S, Shinoda M, Iwata $\mathrm{K}$, et al. Topically injected adrenocorticotropic hormone induces mechanical hypersensitivity on a full-thickness cutaneous wound model in rats. Exp Dermatol 2019; 28 : $1010-6$

19. Zegre Cannon C, Kissling GE, Goulding DR, King-Herbert AP, Blankenship-Paris T. Analgesic effects of tramadol, carprofen or multimodal analgesia in rats undergoing ventral laparotomy. Lab Anim (NY) 2011; 40: 85-93.

20. Roughan JV, Flecknell PA. Behaviour-based assessment of the duration of laparotomy-induced abdominal pain and the analgesic effects of carprofen and buprenorphine in rats. Behav Pharmacol 2004; 15: 461-72.

21. Kabadi R, Kouya F, Cohen HW, Banik RK. Spontaneous painlike behaviors are more sensitive to morphine and buprenorphine than mechanically evoked behaviors in a rat model of acute postoperative pain. Anesth Analg 2015; 120: 472-8.

22. Curtin LI, Grakowsky JA, Suarez M, Thompson AC, DiPirro JM, Martin LB, et al. Evaluation of buprenorphine in a postoperative pain model in rats. Comp Med 2009; 59: 60-71.

23. Cohen J. The effect size index: f. In: Statistical power analysis for the behavioral sciences. 2nd ed. Edited by Cohen J. Hillsdale (NJ), L. Erlbaum Associates. 1988, pp 274-88.

24. Sen CK, Gordillo GM, Roy S, Kirsner R, Lambert L, Hunt TK, et al. Human skin wounds: a major and snowballing threat to public health and the economy. Wound Repair Regen 2009; 17: 763-71.

25. Prevaldi C, Paolillo C, Locatelli C, Ricci G, Catena F, Ansaloni $\mathrm{L}$, et al. Management of traumatic wounds in the Emergency Department: position paper from the Academy of Emergency Medicine and Care (AcEMC) and the World Society of Emergency Surgery (WSES). World J Emerg Surg 2016; 11: 30.

26. Kumar R, Gupta S, Gautam M, Jhajhria SK, Ray SB. Diverse characters of Brennan's paw incision model regarding certain parameters in the rat. Korean J Pain 2019; 32: 168-77.

27. Zahn PK, Gysbers D, Brennan TJ. Effect of systemic and intrathecal morphine in a rat model of postoperative pain. Anesthesiology 1997; 86: 1066-77.

28. Hamalainen MM, Subieta A, Arpey C, Brennan TJ. Differential effect of capsaicin treatment on pain-related behaviors after plantar incision. J Pain 2009; 10: 637-45.

29. Kang SC, Jampachaisri K, Seymour TL, Felt SA, Pacharinsak C. Use of liposomal bupivacaine for postoperative analgesia in an incisional pain model in rats (Rattus norvegicus). J Am Assoc Lab Anim Sci 2017; 56: 63-8.

30. Seymour TL, Adams SC, Felt SA, Jampachaisri K, Yeomans DC, Pacharinsak C. Postoperative analgesia due to sustained-release buprenorphine, sustained-release meloxicam, and carprofen gel in a model of incisional pain in rats 
(Rattus norvegicus). J Am Assoc Lab Anim Sci 2016; 55: 300-5.

31. Castel D, Sabbag I, Meilin S. The effect of local/topical analgesics on incisional pain in a pig model. J Pain Res 2017; 10: 2169-75.

32. Castel D, Naveh M, Aharon A, Doron O, Meilin S. Prolonged analgesic effect of PRF-108 and PRF-110 on post-operative pain in pigs. Pain Ther 2016; 5: 29-42.

33. Wilsey JT, Block JH. Sustained analgesic effect of clonidine co-polymer depot in a porcine incisional pain model. J Pain
Res 2018; 11: 693-701.

34. Cheppudira BP, Garza TH, Petz LN, Clifford JL, Fowler M. Anti-hyperalgesic effects of AG490, a Janus kinase inhibitor, in a rat model of inflammatory pain. Biomed Rep 2015; 3 : 703-6.

35. Golf M, Daniels SE, Onel E. A phase 3, randomized, placebocontrolled trial of DepoFoam ${ }^{\circledR}$ bupivacaine (extendedrelease bupivacaine local analgesic) in bunionectomy. Adv Ther 2011; 28: 776-88. 